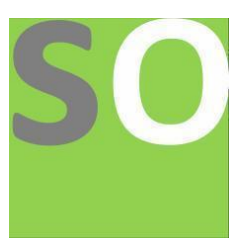

Article title: Mathematical Evaluation of Covid Vaccination Safety and Efficacy

Authors: Amrit Šorli[1], Saeid Jafari [2], Tomaž Makovec [3]

Affiliations: Bijective Physics Institute, Idija, Slovenia[1], Mathematical and Physical Science Foundation 4200

Slagelse, Denmark[2], University of Ljubljana, Medical Faculty, Ljubljana, Slovenia[3]

Orcid ids: 0000-0001-6711-4844[1], 0000-0001-5744-7354[2], 0000-0003-4226-2263[3]

Contact e-mail: amrit.sorli@bistra.si

License information: This work has been published open access under Creative Commons Attribution License http://creativecommons.org/licenses/by/4.0/, which permits unrestricted use, distribution, and reproduction in any medium, provided the original work is properly cited. Conditions, terms of use and publishing policy can be found at https://www.scienceopen.com/.

Preprint statement: This article is a preprint and has not been peer-reviewed, under consideration and submitted to ScienceOpen Preprints for open peer review.

DOI: 10.14293/S2199-1006.1.SOR-.PPAM9MX.v1

Preprint first posted online: 01 February 2022

Keywords: Vaccination, Covid-19, safety, efficacy. 


\title{
Mathematical Evaluation of Covid Vaccination Safety and Efficacy
}

\author{
Amrit Srečko Šorli, physicist, Bijective Physics Institute, Idrija, Slovenia \\ sorli.bijective.physics@gmail.com \\ https://orcid.org/0000-0001-6711-4844 \\ Saeid Jafari, Ph.D. Mathematics \\ Mathematical and Physical Science Foundation \\ 4200 Slagelse, Denmark \\ saeidjafari@ topositus.com \\ https://orcid.org/0000-0001-5744-7354 \\ Tomaž Makovec, mag pharm. Ph.D., University of Ljubljana, \\ Medical Faculty, Ljubljana, Slovenia \\ tomaz.makovec@mf.uni-lj.si \\ https://orcid.org/0000-0003-4226-2263
}

\begin{abstract}
In a given country, we have the set of alive people and the subset of vaccinated people. During a period of 4 weeks, some people that are Covid-19 positive died. Thus, we have another set of Covid-19 positive dead persons that has as its subset Covid-19 positive dead vaccinated persons. We will compare the statistical number of Covid-19 positive dead vaccinated persons with the proportional number of Covid-19 positive dead vaccinated persons, valid in case the vaccines do not affect health. The proportional number must be always bigger than the number of Covid-19 positive dead vaccinated persons provided by official statistical data. In this case, covid vaccines are safe and efficient in saving human lives.
\end{abstract}

Keywords: Vaccination, Covid-19, safety, efficacy.

\section{Introduction}

Alive people in a given country is denoted by the set "alive". In this set, we have a subset called "vaccinated". As people are dying in a given four-week period, we have another set of dead persons. In this set, we have a subset of vaccinated persons. In case vaccines have no impact on death rate, the following equation is valid:

$$
\frac{\text { vaccinated }}{\text { alive }}=\frac{\text { dead vaccinated }}{\text { total deaths }}
$$

We calculate proportional number of dead vaccinated as follows:

$$
\text { dead vaccinated proportional number }=\frac{\text { vaccinated } * \text { total deaths }}{\text { alive }}
$$

The proportional number for a given time period should be always bigger than the statistical number of dead vaccinated. In this case, vaccines are saving lives. 
We will calculate a proportional number of a smaller group of infected people that have been vaccinated and died. The entire set "alive" is exposed to infection with the Sars-CoV-2 virus. According to the official interpretation, vaccinated people are highly protected against infection. Because of this, we expect that the group of infected unvaccinated people will die because of Sars-CoV-2 virus at a much higher rate than the other group of infected vaccinated people. We developed a mathematical model that is evaluating the death rate of the infected vaccinated group and infected unvaccinated group in the set "alive". In both groups, people are dying so we have in a given time period a new set of dead persons that have been infected. Some of them have not been vaccinated and others have been vaccinated.

We have for every 4 weeks a subset of Sars-CoV-2 infected dead people. We assume that vaccination has no impact on people's health, so we will have the same percentage of vaccinated dead persons in the set "alive" as in the set "infected dead".

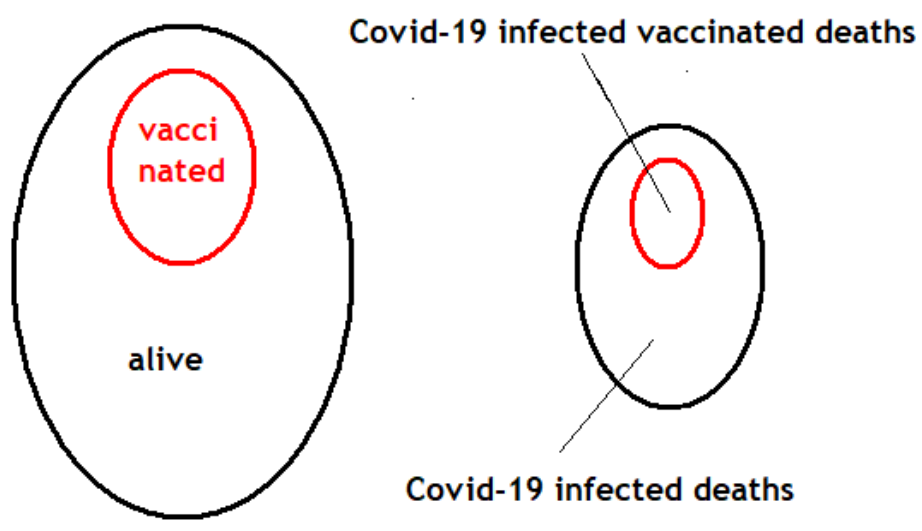

Figure 1: Set of alive and set of Sars-CoV-2 infected deaths

We can write the following equation:

$$
\frac{\text { allive vaccinated }}{\text { alive }}=\frac{\text { Sars }-\mathrm{CoV}-2 \text { infected vaccinated deaths proportional number }}{\text { Sars }-\mathrm{CoV}-2 \text { infected deaths }}
$$

By equation (3) we obtain equation (4):

infected vaccinated deaths proportional $n .=\frac{\text { alive vaccinated } * \text { Sars }-\operatorname{CoV}-2 \text { infected deaths }}{\text { alive }}$

Using equation (4) we can calculate the proportional number of Sars-CoV-2 infected vaccinated dead persons in England in a given 4 weeks. The number we get is a proportional number where there is no impact of the vaccination on the death rate. When $10 \%$ of the population is vaccinated in the group of Covid-9 infected dead persons, $10 \%$ will be vaccinated. When $20 \%$ of the population is vaccinated in the group of Sars-CoV-2 infected dead persons, $20 \%$ will be vaccinated, and so on. When $100 \%$ of the population is vaccinated in the group of Sars-CoV-2 infected dead persons, $100 \%$ will be vaccinated. This "Sars-CoV-2 infected vaccinated dead 
proportional number" (in short "proportional number") can be named "vaccine variant" using terms used in physics.

Now we compare the numbers of Sars-CoV-2 infected vaccinated dead persons in official statistics with the "Sars-CoV-2 infected vaccinated deaths proportional number" we calculate. The statistical number of Sars-CoV-2 infected vaccinated dead persons must be lower than the proportional number. In this case, vaccines are saving people's lives:

$$
\text { covid infected vaccinated deaths }<\text { proportional number }
$$

We can calculate how many Sars-CoV-2 infected persons have been saved from death with vaccination in a given 4 weeks by subtracting the number of Sars-CoV-2 infected vaccinated deaths in statistics from the number of Sars-CoV-2 infected vaccinated deaths proportional number. In case, that the number of Sars-CoV-2 infected vaccinated dead is bigger than the proportional number of covid infected vaccinated dead, vaccination has a negative impact on mortality rate. It takes lives, some people have died prematurely because of being vaccinated.

\section{Methods and results}

\section{The calculation for weeks $35-38$ in year 2021}

A "vaccinated" person in this calculation is the person that was vaccinated with the first dose or more. Let's calculate the proportional number of dead vaccinated in England between weeks 35 and 38. In this timeinterval, there were 3165 covid deaths in the UK. 2448 of them, i.e., ca. $77,35 \%$, have been vaccinated ${ }^{1}$. We see that the percentage of vaccinated people in the UK with one dose in this period is $65.1 \%{ }^{1}$. In 2021 the population of the United Kingdom was 56.191 .000 people. $65.1 \%$ of the entire population is 36.580 .341 people.

$$
\begin{gathered}
\text { proportional number }=\frac{36.580 .341 * 3165}{56.191 .000} \\
\text { proportional number }=2060 .
\end{gathered}
$$

Officially declared vaccinated covid dead persons between 35 and 38 weeks are 2448. This means with absolute growth of $2448-2060=388$ persons and relative growth of $\left(\frac{388}{2060}\right) \cdot 100 \approx$ $18,8 \%$. Therefore 388 persons had prematurely passed away due to the vaccination in the period of weeks 35 - 38 .

\section{The calculation for weeks 39 - 42, 2021}

Let's calculate the proportional number of dead vaccinated in England between weeks 39 and 42. In this period there were 2772 covid deaths in the UK. 2270 of them, i.e., ca. $81,9 \%$, have been vaccinated ${ }^{1}$. We see that the percentage of vaccinated people in the UK with one dose in this period is $66.1 \%^{1}$. The population of the UK was 56.191 .000 people in $2021.66 .1 \%$ of the entire population is 37.142 .251 people. 


$$
\begin{gathered}
\text { proportional number }=\frac{37.142 .251 * 2772}{56.191 .000} \\
\text { proportional number }=1832 .
\end{gathered}
$$

Officially declared vaccinated covid dead persons between 39 and 42 weeks are 2270. This means with absolute growth of $2770-1832=438$ persons and relative growth of $\left(\frac{438}{1832}\right) \cdot 100 \approx$ $23,9 \%$. Thus 438 persons had prematurely passed away due to the vaccination in the period weeks $39-42$.

\section{The calculation for weeks 43-46, 2021}

Let's calculate the proportional number of dead vaccinated in England between weeks 43 and 46. In this period there were 3726 covid deaths in the UK. 2992 of them, i.e., ca. 80,3\%, have been vaccinated ${ }^{1}$. We see that the number of vaccinated people in England in this period with the first dose is $67.4 \%{ }^{1}$. The population of the UK was 56.191 .000 people in $2021.67 .4 \%$ of the entire population is 37.872 .700 people. We use equation (4) and we get:

$$
\begin{gathered}
\text { proportional number }=\frac{37.872 .734 * 3726}{56.191 .000} \\
\text { proportional number }=2511 .
\end{gathered}
$$

Officially declared vaccinated covid dead persons between 43 and 46 weeks are 2992. This means with absolute growth of 2992-2511=481 persons and relative growth of $\left(\frac{481}{2511}\right) \cdot 100 \approx$ $19,2 \%$. So, 481 persons had prematurely passed away due to the vaccination in the period weeks $43-46$.

\section{The calculation for weeks 47-50, 2021}

Let's calculate the proportional number of dead vaccinated in England between weeks 47 and 50. In this period there were 2956 covid deaths in the UK. 2140 of them, i.e., ca. 72,4\%, have been vaccinated ${ }^{1}$. By 19 December 2021, the overall vaccine uptake in the UK for dose 1 was $68.2 \%{ }^{1}$. The population of the UK was 56.191 .000 people in $2021.68 .2 \%$ of the entire population is 38.322.262 people. We use equation (4) and we get:

$$
\begin{gathered}
\text { proportional number }=\frac{38.322 .262 * 2956}{56.191 .000} \\
\text { proportional number }=2016 .
\end{gathered}
$$

Officially declared vaccinated covid dead persons (one dose or more) between 47 and 50 weeks are 2140. This means with absolute growth of 2140-2016=124 persons and relative growth of $\left(\frac{124}{2016}\right) \cdot 100 \approx 6,15 \%$. Thus 124 persons had prematurely passed away due to the vaccination in the period weeks $47-50$.

\section{The calculation for weeks $51,2021-2,2022$}

Let's calculate the proportional number of dead vaccinated in England between weeks 512021 and weeks 2 2022. In this period there were 3893 covid deaths in the UK. 2878 of them, i.e., 
ca. $73,93 \%$, have been vaccinated ${ }^{1}$. By 16 January 2022 , the overall vaccine uptake in England for dose one was $68.9 \%$, see page $3^{1}$. The population of the UK was 56.191 .000 people in 2021. 68.9.\% of the entire population is 38.715 .599 people. We use equation (4) and we get:

$$
\begin{gathered}
\text { proportional number }=\frac{38.715 .599 * 3893}{56.191 .000} \\
\text { proportional number }=2682 .
\end{gathered}
$$

Officially declared vaccinated covid dead persons (one dose or more) between 52 weeks 2021 and weeks 22022 are 2878. This means with absolute growth of 2878-2682=196 persons and relative growth of $\left(\frac{196}{2682}\right) \cdot 100 \approx 7,31 \%$. Hence 196 persons had prematurely passed away due to the vaccination in the period weeks $47-50$ :

Below we have the results of all calculated periods:

weeks 35-38: total covid infected deaths are 3165, number of covid infected vaccinated deaths is 2448 , number of dead because of adverse effects caused by vaccination is 388 which is ca. $15,8 \%$ of covid infected vaccinated deaths.

weeks 39-42: total covid infected deaths are 2772, number of covid infected vaccinated deaths is 2270 , number of dead because of adverse effects caused by vaccination is 438 which is ca. $19,3 \%$ of covid infected vaccinated deaths.

weeks 43-46: total covid infected deaths are 3726, number of covid infected vaccinated deaths is 2992 , number of dead because of adverse effects caused by vaccination is 481 which is ca. $16,1 \%$ of covid infected vaccinated deaths.

weeks 47-50: total covid infected deaths are 2956, number of covid infected vaccinated deaths is 2140 , number of dead because of adverse effects caused by vaccination is 124 which is ca. $5,8 \%$ of covid infected vaccinated deaths.

weeks 51(2021)-2(2022): total covid infected deaths are 3893, number of covid infected vaccinated deaths is 2878 , number of dead because of adverse effects caused by vaccination is 196 which is ca. $6,8 \%$ of covid infected vaccinated deaths.

In 20 weeks, the total deaths of people that were tested covid positive are 16.512. The total number of the covid test positive vaccinated deaths is 12.728 . The total number of deaths because of adverse effects caused by vaccination is 1627 persons. This means in the group of covid test positive vaccinated deaths which is 12.728 persons, i.e., ca. $12.8 \%$ have died because of heavy adverse effects. When all time periods will be calculated, we will have a more detailed percentage.

The total number of deaths of the covid test positive people that died in 20 weeks is 12.728 . The total proportional number of vaccinated infected people that died is 11.101 . This means, in the group of covid positive vaccinated deaths there is a $14.5 \%$ higher rate of mortality than in the group of covid positive unvaccinated considering vaccines would not have any influence on the death rate. Data confirm that infected vaccinated people have a $14.7 \%$ higher probability to 
die of covid infection than infected unvaccinated. When all time periods will be calculated, we will have a more detailed percentage.

According to the official narrative, vaccines are saving lives, which means the number of covid infected vaccinated deaths statistically recorded should be significantly lower than the proportional calculated number of the covid infected vaccinated deaths (see Table 1).

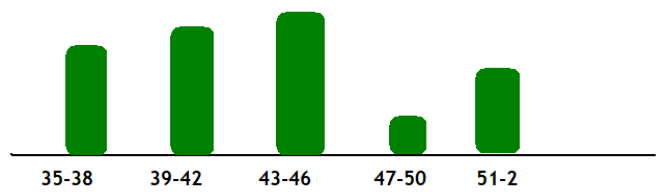

Table 1: Expected results

We got the opposite results. The statistical number of the covid infected vaccinated deaths is significantly higher than the calculated proportional number of covid infected vaccinated deaths, which means that infected vaccinated people are dying at a higher rate than infected unvaccinated, presumably because of negative effects caused by vaccines, see Table 2.

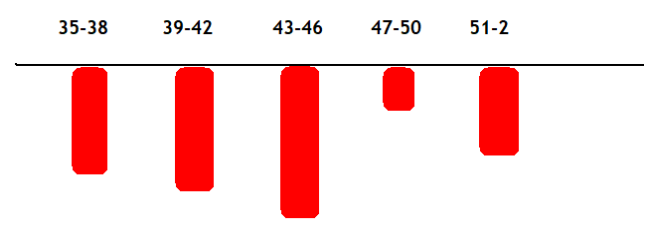

Table 2: Obtained results

Neither would our results be statistically significant if the covid infected vaccinated deaths would decrease in some periods and increase in others (see Table 3).

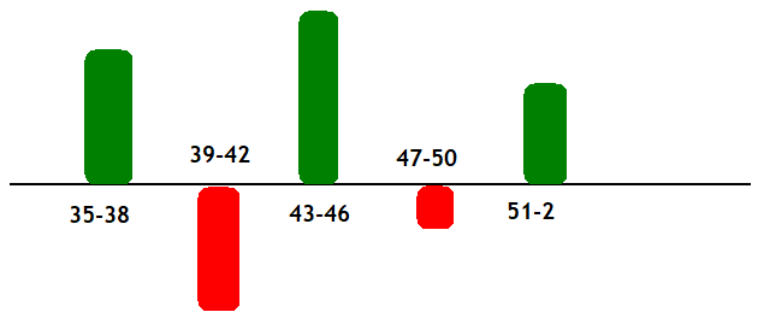

Table 3: Results with no statistical significance

Our results are limited in scope but of high statistical significance. It is unlikely that in any other time period, vaccines would save lives. We do not expect that, had we calculated the 
relationship between the proportional and real number of the vaccinated covid deaths for all time periods, we would get results that contradicted our findings. We are therefore inclined to conclude that covid vaccines are causing adverse effects that lead to premature death, and not only in England also elsewhere.

We believe that the relation between the "vaccinated covid infected deaths" and "nonvaccinated covid infected deaths" in a short time interval of 4 week period is a statistically significant indicator that points to the results of Covid-19 vaccination. Covid vaccination was supposed to be safe and efficient, and it was predicted that it would significantly decrease the number of Sars-CoV-2 infected vaccinated deaths compared to the number of covid infected unvaccinated deaths.

There are no "hidden variables" that could influence the result. Infected vaccinated and infected non-vaccinated people are eating the same food, drinking the same water, breathing the same air, watching the same TV programs. The mathematics of "Sars-CoV-2 infected deaths = SarsCoV-2 infected vaccinated deaths + Sars-CoV-2 infected non-vaccinated deaths" seems to suggest that adverse effects caused by vaccines increase mortality.

\section{Discussion}

The calculations have shown that infected vaccinated people are dying at a 14,5\% percent higher rate than infected non-vaccinated people. The results are confirmed with the numerous adverse reactions of covid vaccination. On the date 19. January 2022 Yellow card is reporting 1.552.248 adverse effects. On the same day 52.168.500 people were vaccinated at least with the first dose. $2.975 \%$ of vaccinated at least with the first dose have adverse effects ${ }^{2}$. It is obvious to conclude that because of some heavy adverse reactions, people are consequently prematurely dying. Other research results report the danger of spike proteins ${ }^{3,4}$ which could also cause deterioration of health and eventually premature death.

At this point, the crucial question is arising: is the Covid-19 vaccine saving more lives or causing excess deaths because of numerous heavy adverse reactions? Research published in NATURE states that the difference in the spread of the virus between those who have been vaccinated and those who have not been vaccinated is negligible ${ }^{5}$. A recent article in LANCET is confirming that Covid-19 is spreading with a similar rate between unvaccinated and vaccinated: "This study showed that the impact of vaccination on community transmission of circulating variants of SARS-Cov-2 appeared not to be significantly different from the impact among unvaccinated people" 6 .

Scientific data are confirming that covid vaccines are not diminishing the spread of the virus and are undoubtedly increasing the death rate ${ }^{5,6}$. At this point, it is clear that further vaccination will not give any positive results. The official narrative is, in 2020 we had excess mortality worldwide because of the Covid-19 pandemic. A belief that massive vaccination against Covid19 is preventing people from dying is false. Yes, people are dying because of Covid-19. But being vaccinated with covid vaccines means the probability of death increases. Our research has shown, vaccinated people are dying at a higher rate than unvaccinated people. This was 
already confirmed by the research of Ingrid Tojersen, namely, covid19 vaccination has been causing the deaths of the elder population ${ }^{7.8}$. Research of Sivan Gazid and co-authors is confirming that natural immunity of unvaccinated people is far better protection than covid vaccines ${ }^{9}$. At this point, it is clear that covid vaccines are not safe and are not efficient; the continuation of vaccination has no necessary scientific confirmation.

BMJ editorial is suggesting, for the rigorous mathematical evaluation of covid vaccines efficacy we need row data ${ }^{10}$. We could calculate the exact impact on the entire population in England and worldwide if we would have data on how many people died in a given time period of four weeks who have been at least once vaccinated. This would allow calculating the proportional number "dead vaccinated" and comparing it with the actual statistical number of deaths. This calculation would give us a result of how massive vaccination decreases the rate of deaths of the entire population preventing people from dying because of covid. Or eventually how massive vaccination increases the death rate of the entire population.

In October 2020 associate editor of BMJ Peter Doshi published a report that current trials are not designed to tell us that the vaccines will save lives ${ }^{11} .15$ months have passed, and we have today the answer. Covid vaccines are not preventing the spreading of Sars-Cov-2" 6 , and natural immunity is better protection than covid vaccination ${ }^{9}$. Our results are showing covid vaccinations are causing adverse effects that have caused infected vaccinated death rate is higher than the unvaccinated death rate.

\section{Conclusions}

Covid vaccination should significantly decrease the number of covid infected vaccinated deaths in comparison to the number of covid infection unvaccinated deaths. There are no "hidden variables" that could influence the results because covid infected vaccinated and covid infected unvaccinated are living under the same conditions. The relation between "Sars-CoV-2 infected unvaccinated deaths" and "Sars-CoV-2 infected vaccinated deaths because of heavy adverse effects" in a short lifespan of 4 weeks is the statistically significant result that Covid-19 vaccines are not safe and not efficient. Our analysis calls for an extensive analysis of Green card data for all four weeks' periods of the time of covid vaccination. In case the results will be the same as obtained for the above four weeks periods, it would be a sign for an urgent call to stop covid vaccination worldwide.

\section{References:}

1. UK HEALTH SECURITY AGENCY https://www.gov.uk/government/publications/covid-19-vaccine-weekly-surveillancereports

2. Yellow cart reports, https://www.gov.uk/government/publications/coronavirus-covid19-vaccine-adverse-reactions

3. Theoharides TC, Conti P. Be aware of SARS-CoV-2 spike protein: There is more than meets the eye. J Biol Regul Homeost Agents. 2021;35(3):833-838.

4. Lei Y, Zhang J, et al. SARS-CoV-2 Spike Protein Impairs Endothelial Function via Downregulation of ACE 2. Circ Res. 2021;128:1323-1326. 
5. Mallapaty S. COVID vaccines cut the risk of transmitting Delta - but not for long, Nature (2021) doi: https://doi.org/10.1038/d41586-021-02689-y.

6. Paredes CF. Transmissibility of SARS-Cov-2 among fully vaccinated. The Lancet 2022;22(1):P16.

7. Tojersen I. Covid-19: Norway investigates 23 deaths in frail elderly patients after vaccination. BMJ 2021;372:n149

8. Tojersen I. Covid-19: Pfizer-BioNTech vaccine is "likely" responsible for deaths of some elderly patients, Norwegian review finds. BMJ 2021;273:n1372

9. Sivan Gazid S, Shlezinger R, et al. Comparing SARS-CoV-2 natural immunity to vaccine-induced immunity: reinfections versus breakthrough infections, August 25, 2021 https://www.medrxiv.org/content/10.1101/2021.08.24.21262415v1.full.pdf

10. Editorials. Covid-19 vaccines and treatments: we must have raw data, now, BMJ 2022; 376:0102

11. Doshi $P$. Will covid-19 vaccines save lives? Current trials aren't designed to tell us BMJ 2020;371:m4037 